\title{
¿Régimen de renta atribuida o semi integrado?: Mayor complejidad y menor equidad
}

René González Madrid*, Magister en Planificación y Gestión Tributaria, Universidad de Santiago de Chile Profesor de Contabilidad y Auditoría, Universidad Alberto Hurtado

Fernando López Gutiérrez**, Ph.D. en Finanzas, Washington University in St. Louis

Profesor de Finanzas, Universidad Alberto Hurtado

La reforma tributaria de 2014 fue una de las principales iniciativas impulsadas por el Gobierno de la presidenta Michelle Bachelet. Los objetivos de esta medida eran aumentar la recaudación tributaria, avanzar en equidad tributaria mejorando la distribución del ingreso, introducir incentivos de ahorro e inversión, así como impulsar medidas para disminuir la evasión y elusión. Entre los principales elementos de esta reforma se encuentra la posibilidad de optar por dos regímenes de declaración de renta que entraron en vigencia a partir del 1 de enero de 2017: régimen de renta atribuida y régimen semi integrado. Este artículo analiza el aumento en la complejidad asociada a este nuevo esquema tributario y las dificultades que conlleva esta elección para pequeños y medianos empresarios. Se propone un periodo de marcha blanca y el desarrollo de herramientas que faciliten la comprensión de las implicancias económicas asociadas a la elección de régimen tributario para los contribuyentes.

Impuesto a la renta pre-reforma

Las personas que son dueñas o tienen participación en la propiedad de empresas que generan ganancias, tienen la obligación de pagar impuestos a través de sus empresas y como personas naturales. Los impuestos que pagan las empresas se denominan de "Primera Categoría" y los impuestos que pagan las personas se denominan "Impuestos Personales" o "Finales". Hasta el 31 de diciembre de 2016, las empresas que debían declarar "rentas efectivas" (nombre técnico que reciben las utilidades para efectos tributarios), lo hacía en un régimen único en el cual todas las empresas pagaban una tasa igual a $24 \%$. Por su parte, los dueños de es- tas empresas debían pagar impuestos personales por las utilidades que retiraban de sus empresas, descontando el $100 \%$ de los impuestos pagados por la empresa cómo crédito contra los impuestos finales. En otras palabras, los contribuyentes propietarios no pagaban impuestos por las utilidades que no retiraban de sus empresas.

Los autores agradecen los valiosos comentarios de los profesores Gustavo Marambio y Nelson Espinoza. Las opiniones y comentarios presentados en este articulo son de exclusiva responabilidad de los autores.

*Email de contacto: rgonzalez@uahurtado.cl

** Email de contacto: felopez@uahurtado.cl 
Regímenes post reforma: Renta atribuida y semi integrado

A contar del $1^{\circ}$ de enero de 2017, la Ley sobre Impuesto a la Renta establece dos nuevos regímenes generales de tributación: el régimen de "renta atribuida" y el régimen de imputación parcial de créditos o sistema "semi integrado". En el primer régimen, las empresas pagarán una tasa de impuesto de $25 \%$ sobre sus rentas efectivas. Por su parte, sus propietarios pagarán impuestos personales por las utilidades atribuidas desde su organización, hagan o no retiro de dichas utilidades. Bajo este régimen, los propietarios pueden utilizar el $100 \%$ del impuesto pagado por la empresa (Primera Categoría) como crédito contra sus impuestos personales. En el régimen semi integrado, las empresas estarán afectas a una tasa de impuesto de $25,5 \%$ por las rentas obtenidas en
2017 y $27 \%$ por aquellas obtenidas a partir de 2018. En este caso, sus propietarios tributarán en sus impuestos personales por las utilidades retiradas y no por las utilidades efectivamente generadas por la empresa. Sin embargo, sólo podrán utilizar un $65 \%$ de los

\section{Cuadro 1}

Comparación de regímenes tributarios

\begin{tabular}{cccc}
\hline & $\begin{array}{c}\text { Régimen } \\
\text { Vigente a 2016 }\end{array}$ & $\begin{array}{c}\text { Régimen de } \\
\text { Renta Atribuida }\end{array}$ & $\begin{array}{c}\text { Régimen } \\
\text { Semi integrado }\end{array}$ \\
\hline $\begin{array}{c}\text { Tasa impuesto pagado } \\
\text { por la empresa }\end{array}$ & $24 \%$ & $25 \%$ & $\begin{array}{c}25,5 \% \text { en 2017 y } \\
27 \% \text { desde 2018 }\end{array}$ \\
\hline $\begin{array}{c}\text { Base de cálculo para } \\
\text { impuestos personales }\end{array}$ & $\begin{array}{c}\text { Utilidad retirada por } \\
\text { los dueños }\end{array}$ & $\begin{array}{c}\text { Utilidad atribuida } \\
\text { (utilidades del ejercicio } \\
\text { atribuibles a una persona) }\end{array}$ & $\begin{array}{c}\text { Utilidad retirada } \\
\text { por los dueños }\end{array}$ \\
\hline $\begin{array}{c}\text { Crédito (rebaja) de } \\
\text { impuestos personales }\end{array}$ & $100 \%$ & $100 \%$ & $65 \%$ \\
\hline Empresas elegibles & Todas & $\begin{array}{c}\text { Sociedades Anónimas y } \\
\text { Sociedades de Personas } \\
\text { con socios que sean } \\
\text { Personas Jurídicas" }\end{array}$ & Todas \\
\hline
\end{tabular}

Fuente: Elaboración propia con datos de Sll y Ministerio de Hacienda. impuestos pagados por la empresa como crédito contra sus impuestos personales. El Cuadro 1 presenta una comparación de las principales características del régimen anterior a la reforma y los nuevos regímenes vigentes con la reforma.

\section{Los empresarios individuales}

Las empresas individuales de responsabilidad limitada (EIRL), las agencias de empresas extranjeras, las comunidades, las sociedades de personas y Sociedades por acciones (SpA) - estas últimas constituidas exclusivamente por personas naturales con domicilio o residencia en el país y/o contribuyentes sin domicilio ni residencia en Chile - tenían la opción de elegir el régimen de tributación general de sus rentas hasta el 31 de diciembre de 2016. El resto de los contribuyentes entre ellas las sociedades anónimas abiertas y cerradas - tributarán por defecto en el sistema semi integrado.

Adicionalmente, aquellos que teniendo la opción de elegir no la llevaron a cabo, la Ley les asigna un régimen por defecto. Empresarios individuales, EIRL, comunidades y las mencionadas sociedades de personas se les asigna el régimen de renta atribuida. A las SpA y agencias se les asigna el sistema semi integrado.

La permanencia en el régimen optado o definido por Ley, será de al menos cinco años antes de poder elegir otra opción. De esta manera, si un contribuyente escoge la opción errada o por defecto se le asigna la opción menos conveniente, podrá tener gravosas consecuencias en la tributación de sus rentas.

\section{Implicancias económicas asociadas a la elección del régimen tributario}

Dependiendo del nivel de renta y el porcentaje de utilidades retiradas por los dueños, la elección del régimen tributario puede tener consecuencias económicas significativas para los contribuyentes. A modo de ejemplo, la Tabla 1 presenta una simulación de la carga tributaria del dueño de una empresa que obtiene una renta liquida imponible (utilidades) de $\$ 100 \mathrm{mi}-$ llones y retira distintos porcentajes de estas utilidades. Se aprecia que cuando al dueño se le atribuye el $100 \%$ de las utilidades (Caso 1), la empresa paga impuestos de primera categoría por $\$ 25$ millones y el dueño recibe una devolución de $\$ 1,5$ millones en sus impuestos personales. Con todo, el contribuyente paga impuestos por $\$ 23,5$ millones. Por su parte, con el régimen semi integrado, con retiro del $100 \%$ de las utilidades, la empresa paga impuestos de primera categoría por \$27 millones y el dueño paga impuestos personales por $\$ 5,9$ millones, con lo cual el contribuyente paga un total de $\$ 32,9$ millones. En este caso, el régimen de renta atribuida produce un ahorro tributario de $\$ 9,5$ millones, cifra equivalente a $9,5 \%$ de la renta generada por la empresa. 
Tabla 1

Comparación de carga tributaria según tasa de retiro de utilidades

\begin{tabular}{|c|c|c|c|c|}
\hline & Atribuido & $\begin{array}{l}\text { Semi } \\
\text { Integrado }\end{array}$ & Atribuido & $\begin{array}{l}\text { Semi } \\
\text { Integrado }\end{array}$ \\
\hline Renta liquida imponible (M\$) & \multicolumn{2}{|c|}{100,000} & \multicolumn{2}{|c|}{100,000} \\
\hline Tasa de retiro de utilidades (\%) & \multicolumn{2}{|c|}{$100 \%$} & \multicolumn{2}{|c|}{$50 \%$} \\
\hline Tasa de Impuesto de Primera Categoría & $25 \%$ & $27 \%$ & $25 \%$ & $27 \%$ \\
\hline Impuesto empresa o 1ª Categoría (M\$) & 25,000 & 27,000 & 25,000 & 27,000 \\
\hline $\begin{array}{l}\text { Base tributaria impuestos personales o } \\
\text { finales }\end{array}$ & 100,000 & 100,000 & 100,000 & 50,000 \\
\hline $\begin{array}{l}\text { Impuesto personal según tabla impuesto } \\
\text { global complementario }\end{array}$ & 23,455 & 23,455 & 23,455 & 5,598 \\
\hline Crédito por impuesto de primera categoría & 25,000 & 17,550 & 25,000 & 17,550 \\
\hline Impuesto personal o final (M\$) & $-1,545$ & 5,905 & $-1,545$ & $-11,952$ \\
\hline $\begin{array}{l}\text { Carga tributaria total ( } 1^{\text {a }} \text { categoría + } \\
\text { personales, M\$) }\end{array}$ & 23,455 & 32,905 & 23,455 & 15,048 \\
\hline Régimen más conveniente & Atribuido & & Semi Inte & ado \\
\hline Ahorro $1^{\mathrm{er}}$ año (M\$) & 9,450 & & 8,407 & \\
\hline
\end{tabular}

Sin embargo, si el mismo contribuyente retira solo un $50 \%$ de sus utilidades (Caso 2), la situación se revierte y el régimen semi integrado le permitirá ahorrar $\$ 8,4$ millones en relación a lo que pagaría con el régimen de renta atribuida durante el periodo'.

En general, el régimen de renta atribuida es conveniente para contribuyentes que retiran la totalidad de las ganancias anuales porque la tasa impositiva es menor y obtienen un mayor crédito asociado a sus impuestos personales que con el régimen semi integrado (100\% versus $65 \%$ ).

Este régimen también le conviene a empresas que perciben un bajo nivel de renta. Por su parte, el régimen semi integrado es conveniente para empresas que tienen proyectado reinvertir sus utilidades en proyectos de largo plazo. En efecto, el menor pago de impuestos aumenta la disponibilidad de recursos para invertir y reduce el costo de financiamiento asociado a fuentes externas.

\section{Limitaciones asociadas a los regímenes de renta atribuida y semi integrado}

Un sistema tributario debería ser simple, flexible para adaptarse a cambios que experimente la economía y generar recursos suficientes para el financiamiento del las obligaciones fiscales. En este contexto, la opción de escoger entre regímenes tributarios aumenta la complejidad de un sistema que ya era considerado complejo. En efecto, la parte de la reforma referente a la elección de regímenes tributarios tiene aproximadamente 16 páginas y la circular elaborada por el Servicio de Impuestos Internos (SII) para orientar su interpretación tiene 209, equivalente a 13 veces el número de páginas de la norma.

Adicionalmente, aunque la elección entre dos regímenes tributarios aumenta la flexibilidad, la obligación de permanecer por cinco años en este la limita. Ya sea por falta de acceso a la información o un nivel de habilidades financieras insuficiente, los errores de elección los pagarán los contribuyentes con menor capacidad para analizar la conveniencia de los distintos regímenes.

\section{Complejidad y capacidad empresarial para elegir el régimen tributario}

Para determinar el régimen tributario más conveniente, los contribuyentes deben proyectar el desempeño financiero, el plan de inversiones y la política de retiro de las ganancias que obtendrá la empresa en un horizonte de cinco años.

Aunque no hay estudios acerca de las habilidades y competencias tributarias de los 1,2 millones de contribuyentes que debían elegir uno de los regímenes disponibles, las estadísticas para la población general sugieren que la formulación y análisis de este problema las sobrepasa enormemente. Por ejemplo, de acuerdo a un estudio de competencias de la población adulta, más de un $80 \%$ de la población chilena tiene serias dificultades para aplicar operaciones aritméticas simples y comprender información escrita (Centro de Microdatos, 2013). En la misma línea, un estudio reciente muestra que menos de $20 \%$ de la población chilena sabe como calcular una tasa de interés simple y menos de un tercio entiende el impacto de la inflación en el valor del dinero (Ormazabal, Sepúlveda y Silva, 2016). Por último, a pesar de las implicancias de la tasa de interés en el costo de los créditos, $46 \%$ de las pequeñas y medianas empresas con ventas anuales superiores a UF800 cotizaron créditos con distintos intermediarios financieros (Arellano \& Peralta, 2015).

(1) Cabe señalar que durante los años siguientes, este contribuyente que retira un $50 \%$ de sus utilidades podría pagar impuestos por las utilidades no retiradas dependiendo de las ganancias y el porcentaje de estas que retire en el futuro. 
Esta cifra aumenta a $60 \%$ para el caso de las microempresas.

Los contribuyentes con bajo nivel de conocimiento financiero tienen al menos dos alternativas para apoyar su análisis del régimen tributario.

La primera es contratar una asesoría contable o tributaria, pero no todos los contribuyentes están dispuestos a pagar por estos servicios ya sea por su costo o por falta de interés. La segunda alternativa es utilizar el simulador del Servicio de impuestos Internos ${ }^{2}$. Sin embargo, esta herramienta presenta dos obstáculos a los contribuyentes.

Por una parte, es necesario que este tenga acceso y esté familiarizado con el uso de internet. Por otra, el simulador se enfoca en el cálculo de la carga tributaria de la empresa pero no permite analizar el pago de impuestos personales bajo distintos escenarios de tasa de retiro de utilidades ni tampoco permite simular la carga tributaria asociada a distintos periodos de tiempo.

Independientemente de los factores que dificultan la elección del régimen tributario, lo concreto es que menos de un $20 \%$ de los contribuyentes que podían optar por un régimen ejerció su derecho a elegir. En la medida que el régimen asignado por defecto sea el más conveniente para los contribuyentes, esto no sería un problema. Sin embargo, es difícil creer que más de $80 \%$ de contribuyentes está conforme con esta asignación.

\section{Propuesta}

A continuación se presenta un conjunto de tres iniciativas que podrían mejorar la implementación de los regímenes tributarios en el contexto de una población con un bajo nivel de habilidades financieras. Primero, permitir a los contribuyentes modificar el régimen tributario seleccionado al 31 de diciembre de 2016. Segundo, durante la declaración de renta del presente año, el Servicio de Impuestos Internos podría informar a los contribuyentes el pago de impuestos que deberían realizar bajo ambos regímenes tributarios, el que eligieron y el que no. Esta información, que se puede entregar de manera escrita o electrónica, ayudará a que los contribuyentes entiendan las implicancias económicas asociadas a la elección los distintos regímenes para sus impuestos personales. Tercero, proporcionar un simulador de obligaciones tributarias asociadas a los distintos regímenes que sea más versátil y facilite el análisis de los efectos tributarios de distintos escenarios de renta y tasa de retiro de utilidades en un horizonte de cinco años.

\section{Referencias}

Arellano, Pamela y Sandra Peralta (2015) Informe de resultados: análisis de financiamiento en las empresas. Tercera Encuesta Longitudinal de Empresas. Ministerio de Economía.

Centro de Microdatos (2013) Segundo Estudio de Competencias Básicas de la Población Adulta 2013 y Comparación Chile 1998-2013.

Ormazabal, F., A. Sepúlveda y N. Silva (2016) Encuesta de medición de capacidades financieras en los países andinos: Chile 2016. Corporación Andina de Fomentoy Superintendencia de Bancos e Instituciones Financieras de Chile.

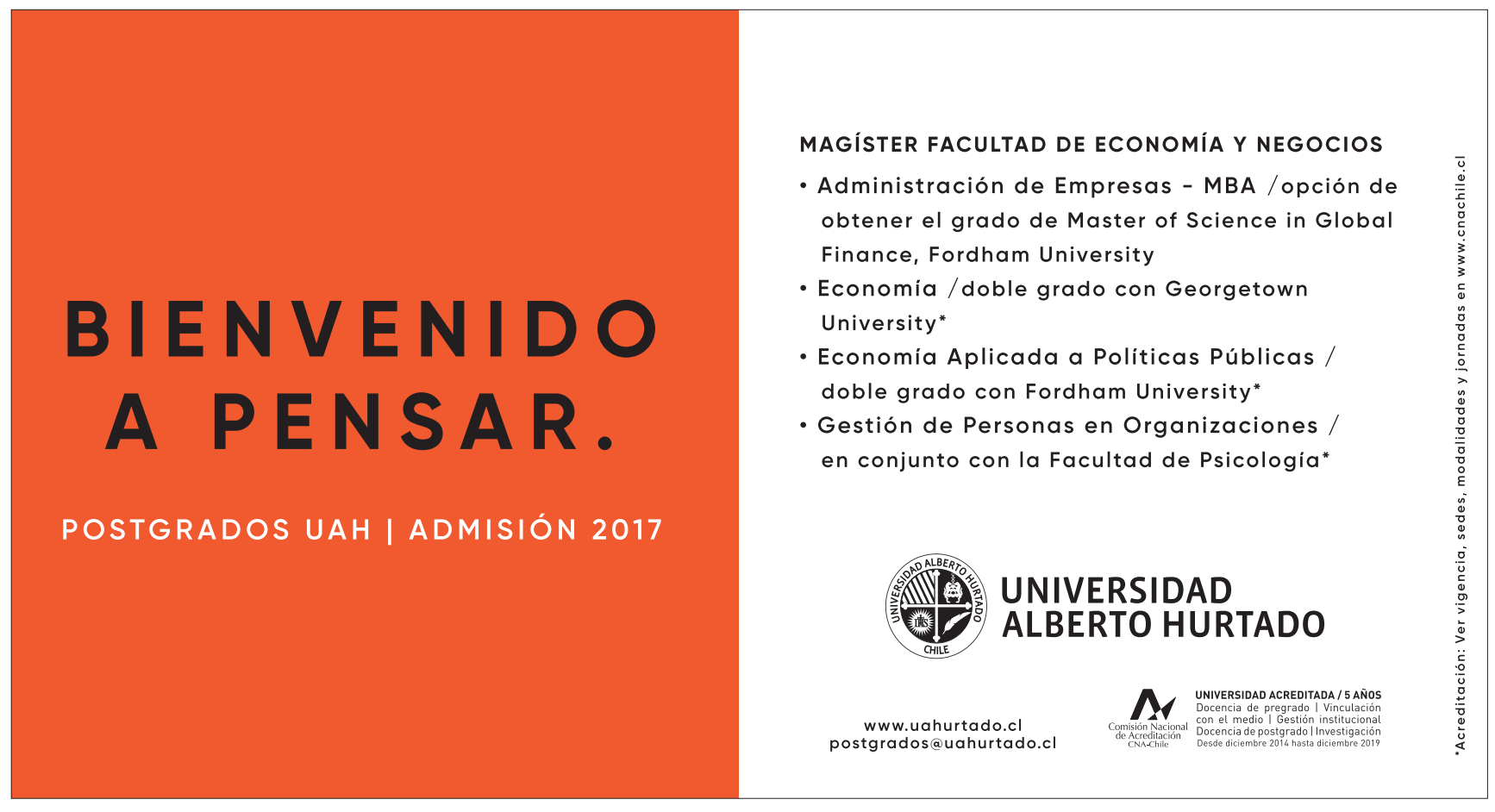

(2) Se puede acceder a través de este vinculo: https://www4.sii.cl/rentaAtribuidalnternet/asistente.html 